\title{
Integrating distributed generation into electric power systems: A review of drivers, challenges and opportunities
}

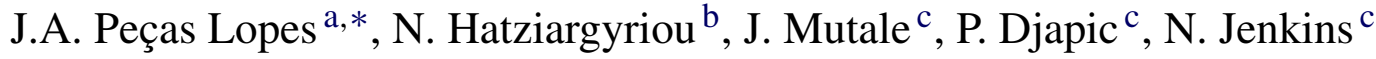 \\ a INESC Porto, Portugal \\ ${ }^{\mathrm{b}}$ NTUA, Greece \\ c The University of Manchester, UK
}

\begin{abstract}
It is now more than a decade since distributed generation (DG) began to excite major interest amongst electric power system planners and operators, energy policy makers and regulators as well as developers. This paper presents an overview of the key issues concerning the integration of distributed generation into electric power systems that are of most interest today. The main drivers behind the focus on DG integration, especially of the renewable type, in many countries around the world are discussed. A synopsis of the main challenges that must be overcome in the process is presented. Particular emphasis is placed on the need to move away from the fit and forget approach of connecting DG to electric power systems to a policy of integrating DG into power system planning and operation through active management of distribution networks and application of other novel concepts. The paper also analyses the repercussions in transmission system operation and expansion that result from the connection of large amounts of DG of different energy conversion systems focusing on issues related with impacts in steady state operation, contingency analysis, protection coordination as well as dynamic behaviour analysis. A discussion on the possibility of provision of ancillary services by DG is also included. Some results from studies performed in the interconnected Portuguese transmission system are presented and discussed. Some of the opportunities that could be exploited in support of the integration and hence greater penetration of DG into electric power systems are also explored. (C) 2006 Elsevier B.V. All rights reserved.
\end{abstract}

Keywords: Distributed generation; Distributed energy resources; Combined heat and power

\section{Introduction}

Although it is now over a decade since distributed generation (DG) became a significant and topical phenomenon in power systems, there is as yet no universal agreement on the definition of DG, which is also known as embedded generation or distributed generation. Current definitions of DG are very diverse and range from $1 \mathrm{~kW}$ PV installations, $1 \mathrm{MW}$ engine generators to $1000 \mathrm{MW}$ offshore wind farms.

Nowadays it is more common for DG to be considered in the context of the wider concept of distributed energy resources (DER), which includes not only DG but also energy storage and responsive loads. The power system architecture of the future, incorporating DER, will look very different from that of today. Whilst the pace of change is likely to be evolutionary, the change itself is expected to be nothing short of a revolution as many

\footnotetext{
* Corresponding author.

E-mail address: jpl@fe.up.pt (J.A.P. Lopes).
}

traditionally held views and approaches to system operation and planning developed over the last 100 years are challenged and transformed to suit the requirements envisaged in the brave new world of the future.

This revolution has already commenced as evidenced by the growth of DG worldwide ([1]; [2]), as Governments strive to achieve ambitious targets of incorporating considerable amounts of distributed renewable generation (DRG) and combined heat and power (CHP) in response to the climate change challenge and the need to enhance fuel diversity. For example, the European Union in the White Book on Reneable Energy Sources (RES), adopted in 1998 ([3] — White Paper for a Community Strategy and Action Plan), has set as target $12 \%$ of electricity supplied by renewable generation by 2010 . According to the 2000 Directive of the European Parliament [4], this is translated to an electricity production of $22.1 \%$ from renewable energy sources. In particular, the progress in wind power development in recent years is impressive. In autumn 2002, almost 27.260 MW of electricity-generating Wind Turbines are operating in fifty countries. Of these, about $75 \%(20.280 \mathrm{MW})$ are 
installed in the European Union, with leading countries covering more than $89 \%$, Germany, Spain and Denmark [5,6]. In addition, considerable developments have been recently made on the technological front, and potentially most challenging in the above respect, is the development of micro-turbines and novel energy storage technologies. The increasing penetration of DG has several technical implications and opens important questions, as to whether the traditional approaches to operation and development of power systems are still adequate. This is particularly true at the distribution level, where the bulk of DG is connected.

This paper discusses the main drivers behind DG growth and presents an overview of the challenges that must be overcome in the integration of DG into electric power systems. In particular, the need to move from the fit and forget policy of connecting DG to electric power systems to a policy of integrating DG into power system planning and operation through active management of distribution networks is emphasised. Some of the opportunities that could be exploited to support the integration and hence greater penetration of DG into electric power systems are also discussed.

The paper also analyses the repercussions in transmission system operation and expansion that result from the connection of large amounts of DG of different energy conversion systems focusing on issues related with impacts in steady state operation, contingency analysis, protection coordination as well as dynamic behaviour analysis. Some results from studies performed in the interconnected Portuguese transmission system are presented and discussed.

\section{Drivers of DG growth}

The primary drivers behind the growth of DG and the current focus on its integration into electric power system operation and planning can be classified into three main categories, namely environmental, commercial and national/regulatory. These drivers are discussed briefly below.

\subsection{Environmental drivers}

\subsubsection{Limiting green house gas $(G H G)$ emissions}

The use of renewable energy and CHP to limit GHG emissions is one of the main drivers for DG. In this regard, it is important to point out that integration of renewable sources of electrical energy into power systems is a somewhat different question from that of the integration of DG into power systems. Integration of DG includes some of the issues related to integration of renewable sources but clearly does not deal with integration of transmission connected renewable sources such as large on shore and off shore wind farms.

\subsubsection{Avoidance of the construction of new transmission circuits and large generating plants}

Another important driver for DG from the environmental perspective is the avoidance of construction of new transmission lines and large power plants to which there is increasing public opposition. There is however also opposition from some envi- ronmental lobby groups to on-shore wind farms on grounds of noise and visual "pollution". There is therefore a balance to be struck between the need for sustainable energy solutions on the one hand and the need to maintain scenic beauty of the environment. Some argue that environmentally benign technologies, such as wind, that do not emit any GHG and have no long term waste management problems should be favoured.

Technological developments in generator technology are already delivering cost effective small to medium size generation technologies for domestic application such as micro-CHP. In times when a premium is placed on land use these technologies are likely to prove popular.

\subsection{Commercial drivers}

\subsubsection{General uncertainty in electricity markets favours small generation schemes}

One of the acknowledged consequences of the introduction of competition and choice in electricity is the increased risk faced by all players in the electricity supply chain from generators through transmission and distribution businesses to retailers. It is well known that the capital outlay required to establish new power stations can be very high. The uncertainties associated with a competitive market environment may favour generation projects with a small capacity whose financial risk is commensurately small.

\subsubsection{DG is a cost effective route to improved power quality and reliability}

The presence of DG close to load centres can have a beneficial impact on power quality and supply reliability. One area of improvement is voltage profile improvements, reduction in number of customer minutes lost especially if DG is allowed and able to stay on when there are network outages (islanding).

\subsection{National/regulatory drivers}

\subsubsection{Diversification of energy sources to enhance energy security}

In recent times, there has been increasing concern amongst energy policy makers regarding energy security. There is a recognition that modern societies have become so dependent on energy resources to the extent that should there be a disruption in its supply the consequences would be too ghastly to contemplate in political, economic and social terms. Because of this the EU energy policy focuses on energy security and sustainability.

In the context of energy security and sustainability, DG is an attractive proposition in many respects. Some of the more important ones are for example:

- It is distributed around the network close to customersfailure of one power station will have limited impact on the whole system compared to failure of one large power plant or bulk electricity transmission facility.

- Diverse technologies and primary energy sources-by diversifying the energy sources especially utilising renewable sources there is sense of control over the nation's future energy 
needs. There is increasing concern that the bulk of fossil fuel based energy supplies come from regions of the world where control of these resources could be potentially unpredictable thus posing an unacceptable risk.

\subsubsection{Support for competition policy}

There is an abiding faith amongst the proponents of reform of electricity supply industries that introduction of competition in generation and customer choice will deliver low energy prices and better service quality. One of the prerequisites for effective competition to occur is that there must be many players in the market. DG clearly advances this cause by providing many small generators that could potentially trade in the energy market and, where appropriate market arrangements exist, also trade in ancillary services. The opportunities for DG to participate in the provision of ancillary services are discussed later in this paper.

\section{Challenges to increased penetration of DG}

Challenges to increased penetration of DG can be classified into three main categories, namely technical, commercial and regulatory. These are discussed in turn below.

\subsection{Technical}

\subsubsection{Voltage rise effect}

The voltage rise effect is a key factor that limits the amount of additional DG capacity that can be connected to rural distribution networks.

\subsubsection{Power quality}

Two aspects of power quality are usually considered to be important: (1) transient voltage variations and (2) harmonic distortion of the network voltage. Depending on the particular circumstance, DG plant can either decrease or increase the quality of the voltage received by other users of the distribution network. Power quality is an increasingly important issue and generation is generally subject to the same regulations as loads. This tends to work well in practice and it is generally possible to meet the required standards by careful design. The effect of increasing the network fault level by adding generation often leads to improved power quality. A notable exception is that a single large DG, e.g. a wind turbine, on a weak network may lead to power quality problems particularly during starting and stopping.

\subsubsection{Protection}

A number of different aspects of DG protection can be identified: Protection of the generation equipment from internal faults; protection of the faulted distribution network from fault currents supplied by the DG; anti-islanding or loss-of-mains protection (islanded operation of DG will be possible in future as penetration of DG increases) and impact of DG on existing distribution system protection. All these aspects are important and need to be carefully addressed in connecting DG to distribution networks.

\subsubsection{Stability}

Traditionally, distribution network design did not need to consider issues of stability as the network was passive and remained stable under most circumstances provided the transmission network was itself stable. Even at present stability is hardly considered when assessing renewable distributed generation schemes. However, this is likely to change as the penetration of these schemes increases and their contribution to network security becomes greater. The areas that need to be considered include transient (first swing stability) as well as long term dynamic stability and voltage collapse.

\subsection{Commercial}

Case studies have indicated that active management of distribution networks can enable significant increases in the amount of DG that can be connected to the existing networks. Although the cost associated with the operation of active distribution networks is still to be identified, it is expected that the benefits are likely to considerably outweigh the cost of its implementation. At present, however, distribution companies that operate wires businesses have no incentives to connect DG and offer active management services. In order to support the development of active distribution networks and extract corresponding benefits associated with connecting increased amount of DG, new commercial arrangements need to be developed.

Generally, three approaches are possible:

- To recover the cost of implementing active management directly through the price control mechanism (increasing the amount of recoverable capital and operating expenditure associated with active management). The cost recovery could be achieved through increased charges for the use of the networks (imposed either to distributed generators benefiting from active management and/or demand customers).

- To establish an incentive scheme that would reward companies for connecting DG, such as one recently developed in the UK [7]. Such an incentive mechanism, assuming a suitable design of the scheme, could lead to the development of active distribution networks. Such schemes could be funded from increased charges imposed on generators and/or demand customers.

- To establish a market mechanism, outside of the regulatory framework, which would create a commercial environment for the development of active networks. Under this scenario, distribution companies would offer active management services to generators for a charge. Clearly, whenever the net benefit from active management exists, this could be used as a basis for bilateral negotiations between the local company and the generator.

The development of active management of distribution networks could stimulate further unbundling of distribution network services with an exchange of services between distribution network operators and distributed generators. 


\subsection{Regulatory}

In the absence of a clear policy and associated regulatory instruments on the treatment of DG, it is very unlikely that this type of generation is going to thrive. The reasons for this are partly historical and related to the way distribution networks have been developed and operated as passive networks. In order to foster the required changes, there is a clear need to develop and articulate appropriate policies that support the integration of DG into distribution networks.

\section{Path forward-active distribution network management}

Active distribution network management is seen as the key to cost effective integration of DG into distribution network planning and operation. This is in direct contrast to the current connect and forget approach.

The historic function of "passive" distribution networks is viewed primarily as the delivery of bulk power form the transmission network to consumers at lower voltages. These networks were designed through deterministic (load flow) studies considering the critical cases so that distribution networks could operate with a minimum amount of control. In other words control problems were solved at the planning stage. This practise of passive operation can limit the capacity of distributed generation that can be connected to an existing system.

For well-designed distribution circuits, there is little scope for distributed generation when simple deterministic rules (e.g. consideration of minimum load and maximum generation) are used. This practice significantly limits the connection of DG. As these conditions may only apply for a few hours per year it is clearly desirable to consider stochastic voltage limits, as proposed under European standard EN 50160. The application of probabilistic load flow [8,9] and Monte-Carlo simulation techniques provide probabilities of voltage limit violations and thus leads to objective decision-making. In Fig. 1, example of a probabilistic load flow analysis is provided [10] showing the probability density

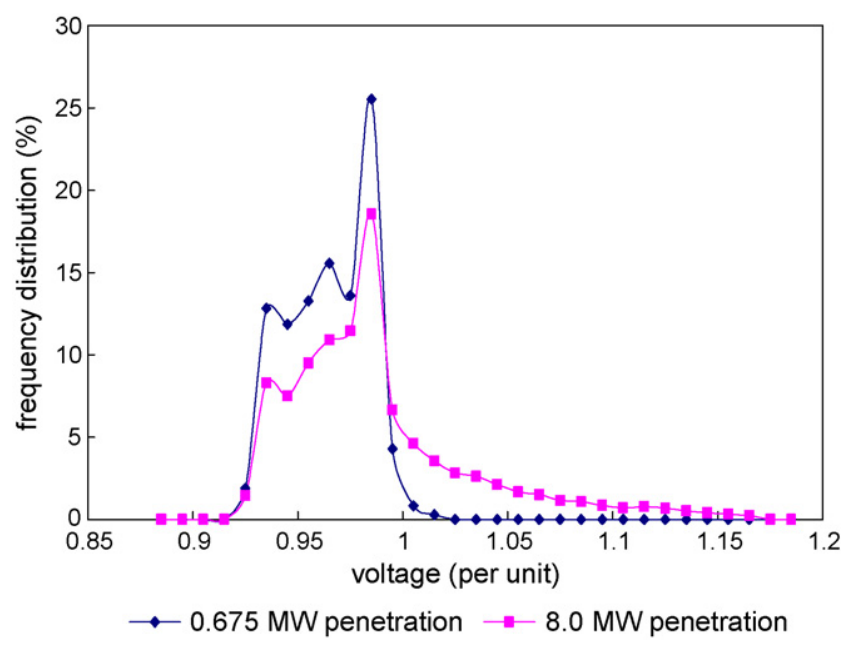

Fig. 1. Impact of wind farm output on the frequency distribution of voltages at node. functions of the voltage at the connection bus of a Wind Park for low levels of installed capacity. For low levels of penetration $(0.675 \mathrm{MW})$, the voltage was generally below 1.0 per unit. However, at higher levels of penetration (8.0 MW), the likelihood of high voltages increased. With a high penetration, the voltage rise effect is very clear (15\% above nominal). It is, however, important to note that whilst the voltage rise is pronounced, the probability of its occurrence is low, less than $6 \%$ in this particular case. Using crude studies, connecting a larger wind farm (8 MW) may have been rejected without considering the likelihood of the increase in voltage. No attention would have been paid to the fact that the mean voltage level over time would be relatively unaffected by the size of the wind farm.

This analysis would also allow distributed generation to decide to be constrained off in certain circumstance to limit voltage rise. Further, many DGs have the ability to operate at various power factors and may even be able to act as sources/sinks of reactive power when not generating. For some overhead distribution circuits (i.e. those with high reactance) then the DGs could contribute to circuit voltage control provided suitable control and commercial systems were in place.

In contrast, active management (AM) techniques enable the distribution network operator to maximise the use of the existing circuits by taking full advantage of generator dispatch, control of transformer taps, voltage regulators, reactive power management and system reconfiguration in an integrated manner. AM of distribution networks can contribute to the balancing of generation with load and ancillary services. In future, distribution management systems could provide real-time network monitoring and control at key network nodes by communicating with generator controls, loads and controllable network devices, such as reactive compensators, voltage regulators and on load tap changing-transformers (OLTC). State estimation and real-time modelling of power capability, load flow, voltage, fault levels and security could be used to make the right scheduling/constraining decisions across the network. These techniques will probably be applied gradually rather than fulfilling all the above listed attributes right from the beginning (Bopp et al., 2003).

The DMS controller software has two functional blocks (see Fig. 2): state estimation and control scheduling. The state estimation block uses the network electrical parameters, network topology, load models and real-time measurements to calculate a network state estimate. The measurement input comprises the local and network measurements. This is passed to the controlscheduling block, which uses it to calculate a new set of control

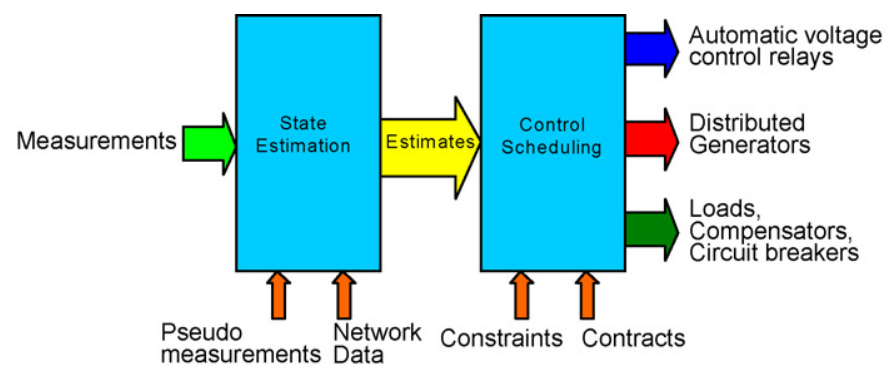

Fig. 2. Block diagram of DMS controller software. 
values for the devices connected to the network. The set of control values optimises the power flow in the network, whilst observing all the constraints and taking account of all the contracts.

The network data input comprises the network topology and electrical parameters. The pseudo measurement input provides values for all un-measured quantities that are required for the state estimator.

The constraints input comprises three types of constraint:

- Primary plant constraints, e.g. distributed generator capacity.

- Control limits, e.g. OLTC maximum number of tap steps.

- Network constraints, e.g. voltage limits.

The contracts input comprises the details of ancillary service contracts between the distribution network operator and owners of:

- distributed generators;

- controllable loads;

- reactive power compensators;

- automatic voltage control relays.

Coordinated voltage control with on load tap changers and voltage regulators generally leads to operating arrangements requiring a number of measurements from key network points as well as communications.

From a regulator's perspective, active management should enable open access to distribution networks. It has the function of facilitating competition and the growth of small-scale generation. In addition, the use of the existing distribution assets should be maximised to minimise costs to consumers.

Therefore, an integral understanding of the interrelated technical, economic and regulatory issues of active management and DG is important for the development of the future distribution systems [11].

\section{Ancillary services from DG}

As DG penetration increases it will become an economic imperative that DG participates in the provision of ancillary services needed for secure and reliable operation of the power system. This is important for the simple reason that if DG only displaces the energy produced by central generation but not the associated flexibility and capacity, the overall cost of operating the entire system will rise.

Another reason for exploring ancillary service provision by DG is to improve the economic viability of some DG projects.

There is potential for distribution network ancillary service markets to develop in-line with the anticipated increase in electricity generation from distributed resources. A study was conducted to investigate this potential [12]. The study sought to evaluate the distribution ancillary service market opportunities applicable to both renewable and non-renewable forms of distributed generation.

A pre-requisite for the detailed development of operational and commercial models was that any new services should be financially material to the distributed generator whilst remaining economically and operationally attractive to network operators. Consequently, value based approaches were adopted for each ancillary service with a view towards improving the attractiveness of distributed and renewable generation projects. In order to demonstrate the application of the proposed approach the UK electricity market was used as a case study partly because it is one of the most advanced competitive markets in Europe and provides practical examples of the issues under investigation.

The services for which potential arrangements were explored are:

- TSO frequency response;

- TSO regulating and standing reserve;

- TSO reactive power;

- DNO security of supply contributions;

- DNO quality of supply services;

- DNO voltage and power flow management services.

\subsection{TSO frequency response}

Frequency response services are required by the TSO to maintain the system frequency within statutory tolerances. A key feature of TSO frequency response provision is the requirement for generators to be part loaded. It is unlikely that TSO frequency response services will be provided regularly by renewable generation, as the rewards from providing such services would be insufficient to compensate for losses of ROC (Renewable Obligation Certificate, a green energy premium) and energy revenues. Distribution connected combined cycle gas turbine (CCGT) plant already provides this service to TSOs.

Infrastructure and generator size considerations suggest that frequency response services will remain attractive to large, non-renewable generators. An innovative, low-cost means of scheduling automated mass responses from highly flexible small plant can be developed. If such a scenario does arise, it is likely that the scope for aggregation services will increase.

Although mandatory frequency response capabilities may become a technical requirement for large distribution connected wind farms, thereby 'resolving' any infrastructure constraints, the extent to which the TSO will utilise such capabilities is unclear. The attractiveness to wind operators, of regularly providing such services is similarly unclear due to the loss of ROC revenue.

The value of TSO frequency response is estimated to vary between $€ 0.59 / \mathrm{kW}$ per annum for wind generation and $€ 3.72 / \mathrm{kW}$ per annum for CCGT technology.

\subsection{TSO regulating and standing reserve}

Reserve energy is required to provide rapid access to generation, to accommodate errors in demand forecasting, to provide contingency arrangements for generation failures and to restore frequency response capabilities.

The key differences between frequency response and reserve services relate to delivery timescales. Typically, reserve services 
are manually initiated and involve longer lead times. A consequence of simplified service initiation procedures is a reduction in the sophistication of control requirements, thus making reserve more attractive to smaller providers.

It is unlikely that synchronised reserve will be provided by renewable generation, as the compensation for the loss of ROC revenue (green energy premium) would be insufficient to justify part load operation. Non-renewable distributed generation already provides standing reserve services to the TSO at a value of approximately $€ 10.4 / \mathrm{kW}$ per annum. Increased DG participation could be facilitated by expanded aggregation services.

\subsection{TSO reactive power}

TSO reactive power can be sourced from distributed generators, especially those connected at $132 \mathrm{kV}$, for transmission system voltage regulation. Reactive power sourced at lower distribution voltages will effectively displace reactive power from transmission-connected generation.

DG connected at lower voltage levels can make a significant impact on the amount of reactive power exchanged between TSO and DNO systems. A simple generic model was developed to illustrate DNO reactive power import reductions at different levels of DG penetration. The value of DG derived reductions in DNO reactive imports was estimated to be approximately $€ 1.78 / \mathrm{kW}$ per annum. The impact of DG on TSO reactive power market will be driven by many different variables. More work would be required to determine the impact of DG on DNO reactive requirements.

The impact of reactive power management on the transport capabilities of distribution circuits was also investigated. DG connected close to loads could extend the transport capabilities of existing circuits. The value of this service would be limited by the low cost of power factor compensation equipment. It is unlikely that this would represent significant income for DG. High DG availabilities would be needed for DNOs to consider such services.

\subsection{DNO security of supply contributions}

The proposed planning recommendation ER P2/6 in the UK could broaden opportunities for DNOs to consider contributions to network security from DG. However, as most DNO networks are largely ER P2/5 compliant, the requirements for security contributions from DG may be limited in the short-term. In the medium to long term, load growth and asset replacements could increase opportunities for DG to provide network support services.

The value of security provided by non-intermittent DG can be related to the avoided or deferred costs of network reinforcement. DG can also substitute for network automation facilities. This is particularly relevant when considering security contribution of intermittent generation such as wind.

A number of examples were used to illustrate the potential value of network security services. For non-intermittent generators, values in the range of $€ 1.49-17.83 \mathrm{~kW}^{-1}$ per annum were derived, depending upon the complexity of the network solution.
It is anticipated that most reinforcements would be at the lower end of this range.

Because of the drive to reduce customer interruptions (CI) and customer minutes lost (CML), DNOs in the UK have made considerable investments in 11 and $0.4 \mathrm{kV}$ networks. A result of this investment is that distribution networks in UK are generally "over compliant" with planning and security standards. For the foreseeable future, the scope for DG to provide security services could be limited.

\subsection{DNO quality of supply services}

In the future, there could be opportunities for DG to improve service quality on 11 and $0.4 \mathrm{kV}$ networks, given the contribution of such networks to quality of supply statistics. In order for DG to improve service quality on such networks, the generation must also be connected at 11 or $0.4 \mathrm{kV}$, thus restricting opportunities to relatively small sized generation. A key requirement for DG, to reduce the impact of outages, is islanded operating capability.

Analysis suggests that the annual benefit of islanding operation was approximately $€ 2.08$ and $€ 28.23 \mathrm{~kW}^{-1}$ per annum for residential and commercial customers, respectively. Due to the complexity of islanding, it is unlikely that DG will be able to significantly reduce CIs and CMLs in the short or medium term.

\subsection{DNO voltage and power flow management services}

Analysis revealed that voltage control and flow management problems are essentially network planning related issues as they relate to supply restoration times following network failures (in the context of UK security standards ER P2/5 or P2/6). The focus here is in generators providing services critical to system restoration (e.g. voltage support or flow control) after a fault occurs rather than under normal operating conditions when the network will tend to provide services to DG in order to maximise DG output (e.g. tap changing and flow control).

Because of the relatively low availability of DG compared to network components and the UK's deterministic voltage standards, opportunities will be limited for DG to provide voltage support or overload reduction. Only non-intermittent DG would be suitable for such applications. Opportunities will improve with increased DG penetrations due to the higher collective availability. The value of these services was estimated to be of the order of $€ 2.23 / \mathrm{kW}$ per annum.

\subsection{Ancillary service capabilities of different generation technologies}

Whilst all of the above services were explored in detail, only TSO frequency response, TSO regulating and standing reserve and DNO security of supply contributions represent realistic opportunities for distributed generators in the short or medium term.

Combined cycle gas turbines (CCGT) and DFIG wind generators were the most promising technologies for the provision of TSO frequency response services whereas CCGTs, diesel standby generators and perhaps micro-CHP were best 
placed to provide reserve services. Synchronous wind generators connected to the grid through power electronics interface also present good capabilities for frequency control. Moreover due to pitch control, some squirrel-cage induction wind generator may also contribute (in a limited way) to frequency control.

It was found that, to varying degrees, DNO security of supply services could be provided by most existing distributed generation technologies.

As the majority of existing DG has been installed for electricity supply purposes, very few generators are equipped with the infrastructure necessary to provide ancillary services. Such infrastructure includes governors, automatic voltage regulators, resynchronisation facilities, and appropriate protection, monitoring and communication facilities.

The most appropriate commercial arrangements for response and reserve services appear to be market-based mechanisms. Ideally the TSO's current arrangements could be extended. Expanded aggregation arrangements, utilising lower cost infrastructure, would facilitate increased participation from small generators.

The most appropriate commercial arrangements for DNO security of supply services appear to be bilateral contracts due to the local and site-specific nature of security requirements. A methodology for pricing distribution network use that recognises the contribution of DG to security has been illustrated.

Opportunities for DG to provide ancillary services will undoubtedly increase as DG penetrations and availabilities increase.

The analysis undertaken suggests that value of the most feasible ancillary services will be relatively low. Consequently, such services will represent incremental revenue opportunities for DG. In general, it would not be possible to develop business cases for investing in DG solely on the basis of ancillary service income.

Niche opportunities will emerge for DG to provide ancillary services, usually in circumstances where constraints restrict network development, e.g. environmental, planning and terrain related constraints.

In an era with significantly increased levels of DG operating on active distribution networks, the opportunities for DG to provide ancillary services may increase. However, on active networks there is an increased likelihood that due to distribution network constraints, certain modes of operation may not be permitted by the DNO. Consequently, there could be increased delivery uncertainty regarding the provision of TSO ancillary services from distributed generators connected to active networks.

In circumstances where a distributed generator receives conflicting instructions regarding the provision of different ancillary services, local services should take precedence over national services.

Higher penetrations of DG will increase DNO options regarding network operation and development decisions, which could lead to lower overall costs.

Increased penetration of DG could also enhance competition in TSO markets for frequency response and reserve. This could be particularly relevant should demand for these services increase with intermittent generation.

In the UK energy market under new electricity trading arrangements (NETA), the output from distributed generation is largely purchased by suppliers and settled through supplier demand accounts. Therefore, suppliers must ensure they are aware of generator operating regimes and also whether generator operation is likely to be influenced by ancillary service provision. Supplier concerns will relate to imbalance exposures in the BM and the fulfilment of ROC targets. Suppliers will require notification of ancillary service provision, in order to suitably revise demand forecasts. Ancillary service instructions issued post-Gate Closure will inevitably impact upon a supplier's imbalance exposure and potentially reduce the value of the energy supplied.

It is important to stress that provision of ancillary services from DG will not jeopardise or degrade security of supply but should contribute to its enhancement.

\section{Technical impacts of DG on transmission system operation}

The technical impacts of DG in system operation can be evaluated at different levels: (a) steady state operation; (b) protection coordination; (c) dynamic behaviour (with a natural interaction with the protection solutions adopted, namely from the DG side); (d) provision of ancillary services.

Evaluations of the impacts in the transmission network involve a large number of studies, considering different typical scenarios of operation (for instance peak load hours, valley hours and mid consumption levels). In power systems where there is a considerable contribution from hydropower, the number of these scenarios increases since it will be necessary to evaluate impacts for winter (wet or dry) and summer periods. The intermittent nature of some of DG plants (such as wind parks) can also lead to the need to consider additional scenarios with different levels of DG production.

These impacts can be analysed by comparing the solutions obtained for each scenario before and after the introduction of the DG levels for a given study. Two types of analyses can be conducted:

- Global evaluation of system performance.

- Analysis of the electrical behaviour in specific network areas, due to specificities of the energy conversion systems adopted and due to the characteristics of the local network.

\subsection{Steady state operation}

Steady state operation impacts are evaluated in terms of changes in voltage profiles, active and reactive losses and congestion levels in system branches through the solution of load flow problems. A change in the amount of DG level also requires a previous power dispatch in order to allocate to the conventional units the power that needs to be produced for each scenario to be considered. Assuming that the total DG production level can be defined as $P_{\mathrm{DG}}$ and the total consumption level is $P_{\mathrm{L}}$, the 


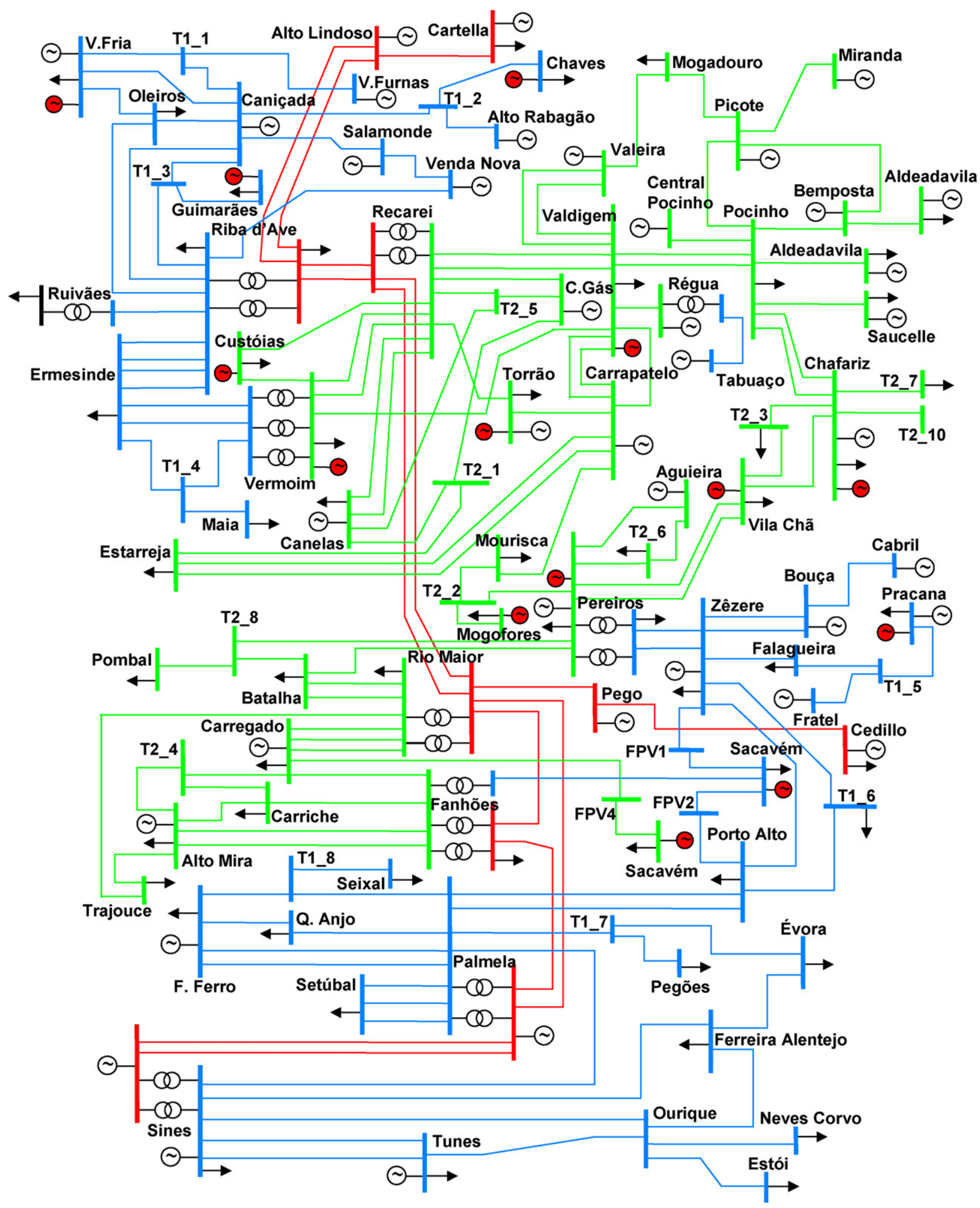

Fig. 3. Single line diagram of the Portuguese transmission system in 2000.

amount of power to be produced by the conventional units $\left(P_{\mathrm{G}}\right)$ is:

$P_{\mathrm{G}}=P_{\mathrm{L}}+P_{\text {losses }}-P_{\mathrm{DG}}$

This means that the steady state impacts are largely dependent on the final dispatch solution to be adopted.
An example of the type of results obtained from this type of steady state impacts studies is presented next, through the results derived from the analysis performed in the Portuguese interconnected transmission grid, described in Fig. 3 (Peças Lopes, 2002). Three transmission levels were taken into account in this study: 150,220 and $400 \mathrm{kV}$. The three interconnections corridors 


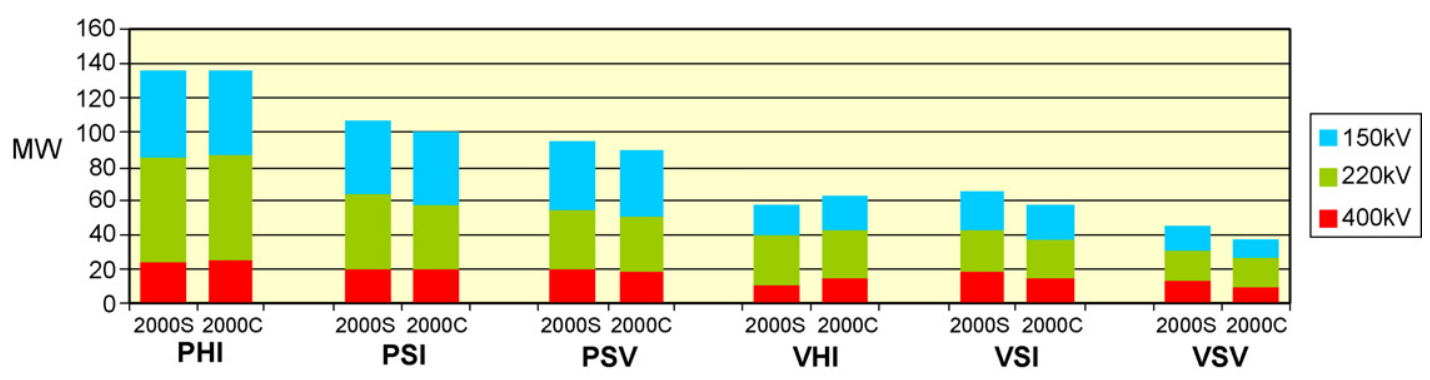

Fig. 4. Changes in transmission active losses for the six different scenarios.

with Spain were also considered in this analysis, by keeping the usual import/export plan on each of the analysed scenarios.

The impact evaluation study was conducted in order to analyse the influence of an additional connection of about $300 \mathrm{MW}$ of DG in the north part of Portugal, without reinforcing the existing network. An amount of $1300 \mathrm{MW}$, with large participation of cogeneration, was considered to be already in operation.

The Portuguese system is characterized by a considerable amount of hydro production in the north and a large concentration of thermal production in the area of Lisbon and south of Lisbon. Six scenarios were therefore considered for this analysis: peak load wet winter (PHI), peak load dry winter (PSI), peak load dry summer (PSV), valley hours during wet winter (VHI), valley hours during dry winter (VSI) and valley hours during dry summer (VSV). For the solution of the load flow problem at the transmission level, DG plants can be modelled as injections of active and reactive power. This modelling approach, adopted in the described study, results also from the fact that, in Portugal, these plants are obliged to follow a generation rule such that they have to produce, during the off-valley hours, reactive power in the amount of $40 \%$ of the active power, and they should not inject reactive power in the grid during the valley hours. However, in the case of large concentration of induction generators a different bus bar model needs to be considered in order to tackle the impacts of voltage on active and reactive power outputs of the machines. The R, X model (or special P, Q model) described in Ref. [13] can be used to provide more accurate results from these types of studies, and to account for near to voltage collapse operating conditions.

In network areas with large concentration of wind generation using asynchronous machines, large voltage excursions may happen also as a result of the amplification effect in reactive power produced by shunt capacitor banks used to generate the needed reactive power, which varies with the square of the voltage [14]. In these areas if an excessive low voltage takes place, it may cause wind generators and customer induction motors to stall, provoking a voltage collapse.

The results obtained in the described system, with the normal topological configuration, show that, as expected in a transmission system with such a meshed structure, there were no large changes in voltage profiles, since the transmission grid has a considerable capacity of controlling voltages by exploiting OLTC transformers and synchronous generators in the main power stations. This was verified although there was a considerable amount of reactive power injected by the DG plants into the grids during the off-valley hours, which contributes to the reactive power support, but is regulated according to the needs of the system.

Variation in active losses in the studied system can be observed in Fig. 4. These changes are described for the six different scenarios, before (2000 S) and after (2000 C) the inclusion of the DG output.

From Fig. 4, it is observed that there is a small increase in active losses for the wet scenarios and a decrease in losses for the dry scenarios. This results from the fact that, in this network, the large concentration of DG coincides geographically with the big hydro generation area.

However, if the increase in DG takes place in industrial areas, clearly a reduction in active losses occurs with consequent large economic benefits that are added to the fact that thermal production is reduced in proportion to the amount of the DG power delivered to the network.

In any network, branch loadings are increased or decreased depending on the production level of the conventional plants. In the situation analysed for the Portuguese network where there is a large concentration of DG in rural areas, two different cases were detected:

- Wet periods, where there is an increase in branch congestion levels connecting the main hydro production areas, in the north, and the north-south transmission corridors;

- Dry periods, where there is a clear reduction in branch loading, since the contribution of DG production (concentrated in the north) decreases the usual south-north power flows.

If DG production is concentrated in the large urban and industrial areas the congestion levels decrease in most of the branches, for all the conventional production scenarios, which is an important technical and economical benefit.

A complementary steady state analysis can be performed for each scenario through a probabilistic load flow or a fuzzy load flow, where the DG productions are replaced, respectively, by probabilistic distributions or fuzzy distributions, namely when there is not enough statistical data do built a probabilistic model, according to the specific characteristics of the primary energy resources. Such an analysis will produce, instead of deterministic values, a set of distributions for the power flows in the transmission lines and for the voltages in the system buses. This will provide a complete picture about the impacts in the network resulting from the variability of the DG resources [15]. 
From this analysis, one can conclude that the impacts on the steady state operating conditions of the transmission network are strongly dependent on the consumption levels of the distribution side, producing clear benefits for the grid when DG is connected in large consumption areas and creating some problems (specially overload situations) for those scenarios where the DG production coincides with the production from large conventional units, situated in low load density zones.

\subsection{Contingency analysis}

The impacts from the connection of DG on $(n-1)$ contingency analysis need also to be addressed, since the increase in branch loadings may create unexpected overload problems in some branches after a given contingency. In fact, for the usual operation strategy, the system may be considered as secure from the steady state point of view and become insecure after the connection of DG in the network, because these new injections will be responsible for some the overloads that may occur.

This issue may become the limiting factor to the increase of DG production in areas with low load consumption levels. Contingency analysis can therefore be performed in a way that it can be used to identify the maximum allowable DG production levels in export areas. For that purpose a sensitivity analysis regarding load congestion in affected branches, relatively to the increases of DG injection in system buses, can be performed in the postcontingency network to identify the degree of responsibility of these productions in branch overloads.

When those branch overloads are detected, this means that line up grade or construction of new additional lines are needed in these network areas. In order to assure a robust transmission system, the connection to large wind farms requires also, as for conventional generation plants, enough redundancy. A discussion on who should pay for the system reinforcements can then be developed. Either the transmission system operator pays for those investments, recovering these costs in the tariffs, according to a general energy policy of the country related with the promotion of renewable energies, or the promoters of the DG plants share these costs amongst them in some way.

Such an impact evaluation approach needs to be developed in carefully, since, if this contingency analysis is conducted in a deterministic manner for a scenario of simultaneous nominal or near nominal DG production level, it will produce serious limitations to the integration of DG in those power export areas. Such coincidence in DG production will rarely take place, and if this happens the right approach should be to allow generation curtailment, for a limited period of time. This will enable the connection of a large amount of DG capacity and may be particularly suitable for wind generation, as this generation curtailment is likely to be required during only short periods of time.

\subsection{Protection schemes}

Apart from own protection schemes that each generator should possess [11], DG plants are often required to install a set of protection systems for interconnection with the local grid, to ensure that the production plant will be disconnected from the network once a fault is detected in the grid. In Portugal, this set of protection-schemes includes three phase overcurrent relays, three phase under and over voltage relays, under and over frequency relays and a zero sequence voltage relay (used to detect in an efficient way impedance earth faults in the distribution network). Usually these relays are set to instantaneous operation, due to the need to assure the safety of maintenance staff.

In a scenario of large participation of DG, concentrated in one area, a simultaneous miss operation of the interconnection relays of these DG plants may provoke serious system operation problems. In fact, if a short-circuit takes place in the transmission grid and its effects on voltage are propagated downstream to the distribution level, it may provoke the disconnection of a large amount of DG production. Since these DG plants are tripped, a sudden increase in power flows coming from upstream, takes place in this network area, such that it may provoke cascading operation of overcurrent relays in distribution and transmission branches, as a result of overload situations. Furthermore, the voltage profiles in the area where the DG was disconnected can be seriously affected and initiate a voltage collapse phenomena. In the next section, some of the consequences of such an incident are presented and analysed in terms of impacts on dynamic behaviour of the system.

This means that coordination in time delays between the system protection strategy of the transmission grid and the time delays of the voltage interconnection protections of the larger DG plants (that must have a different regulation than $0 \mathrm{~s}$ ) must be studied and implemented. The philosophy of such coordination should be:

$T_{\text {tg }}<T_{\mathrm{DG}}<T_{\text {rec }}$

where:

- $T_{\mathrm{tg}}$ - the largest fault elimination time for a short circuit in the transmission grid.

- $T_{\mathrm{DG}}$ - time delay of the interconnection protection of the DG plants.

- $T_{\text {rec }}$-reclosure time in MV feeders at the MV outputs of the HV/MV substation (usually $300 \mathrm{~ms}$ ).

However, this approach should never compromise, in any way, the selectivity of the protections between the transmission and distribution networks and the selectivity of protections within the distribution grid itself.

\subsection{Dynamic behaviour}

The increase in the share of DG production in electrical networks is more and more requiring an analysis of the system dynamic behaviour of some incidents that may occur. These studies should be developed carefully taking the following into consideration:

- DG dynamic modelling according to the energy resource and adopted conversion systems;

- aggregation of DG generating units according to their type;

- definition of a set of critical disturbances. 


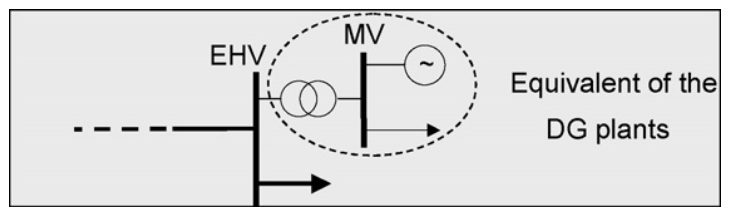

Fig. 5. Representation of equivalent DG units.

From the transmission point of view and for this analysis, the DG units should be aggregated according to generation type, such that they could be represented as shown in Fig. 5.

\subsubsection{DG modeling}

A proper dynamic modelling of the DG units, for dynamic behaviour studies, is a key issue to get an adequate idea of the impact in the network resulting from the presence of these generation units following some disturbances. In such dynamic analysis one must remember that only low frequencies phenomena are of interest.

Synchronous machines should be modelled through the conventional state equations that describe the electrical machine, automatic voltage regulator, swing equation, speed regulator and primary machine [16]. This modelling depends however on the type of plant. Usually mini hydro plants do not have speed regulation. This is also often the case with cogeneration plants, where the speed regulator is not activated when operating in grid connected mode. In some industrial plants that have cogeneration facilities the speed regulator becomes active only in isolated operation mode.

Asynchronous generators can be modelled in a proper way, for grid dynamic behaviour analysis, through a third order model with one mechanical equation and two electrical equations, as described in [13]. Generators with power electronic converters can be modelled as current controlled sources [13] or active and reactive power sources [17] since the fast transients in these converters, related with electronic switches operation and control, are of no interest in such analysis. The aggregation of synchronous and asynchronous machines can be made adopting the usual procedures used for the aggregation of conventional units in per unit $[16,18]$, neglecting the distribution grid connections situated downstream. Generators with power electronic interfaces can be modelled as mentioned in the previous paragraph, assuming an equivalent power output equal to the sum of the individual outputs of each one of these units [19]. An important issue to be mentioned is that machines with full grid electronic interfaces, characterized by no inertia, may become a problem regarding stability issues.

\subsubsection{Critical disturbances}

Having in mind the characteristics of the DG units namely the fact of not participating in frequency regulation, the type of relay settings imposed to the interconnection protection and the intrinsic nature of the power electronics interface of static generators, that are quickly disconnected from grid when large voltage drops occur, some of the most critical disturbances to be considered are:
Table 1

Characterisation of disturbances

\begin{tabular}{lll}
\hline & Disturbance 1 & Disturbance 2 \\
\hline Characterisation & $\begin{array}{l}\text { Disconnection of one of } \\
\text { the interconnection with } \\
\text { Spain (150 MW import) }\end{array}$ & $\begin{array}{l}\text { Short circuit in one 220 kV } \\
\text { transmission line near a } \\
\end{array}$ \\
& $\begin{array}{l}\text { substation in the north of } \\
\text { Portugal, eliminated after } \\
100 \text { ms, provoking the } \\
\text { disconnection of 500 MW } \\
\text { of DG production }\end{array}$
\end{tabular}

Scenario 1

$500 \mathrm{MW}$ of DG

production for a wet peak load period

Scenario 2 $1500 \mathrm{MW}$ of DG

$1500 \mathrm{MW}$ of DG production production for a wet peak for a wet peak load period load period

- short circuit in the transmission grid;

- loss of an important conventional unit or loss of an important interconnection line.

\subsubsection{Dynamic behaviour}

In order to illustrate the type of impacts that some disturbances in the transmission grid can produce in the system behaviour, results obtained for a generation scenario of an equivalent of the Portuguese/Spanish interconnected system are described next. The simulated disturbances are summarised in Table 1. The DG units considered in these simulations were about $70 \%$ of synchronous machines (with low inertia) and 30\% of asynchronous ones, distributed all over the Portuguese side of the network, with a larger concentration in the north part of the Portuguese system. The mechanical power of these generating units was kept constant and equal to the pre-fault values during all the simulations.

In Fig. 6, the frequency behaviour of the system is depicted. Fig. 7 describes the dynamic behaviour of the power flow in one of the $400 \mathrm{kV}$ remaining interconnections for both DG production levels. The solid line corresponds to the system behaviour

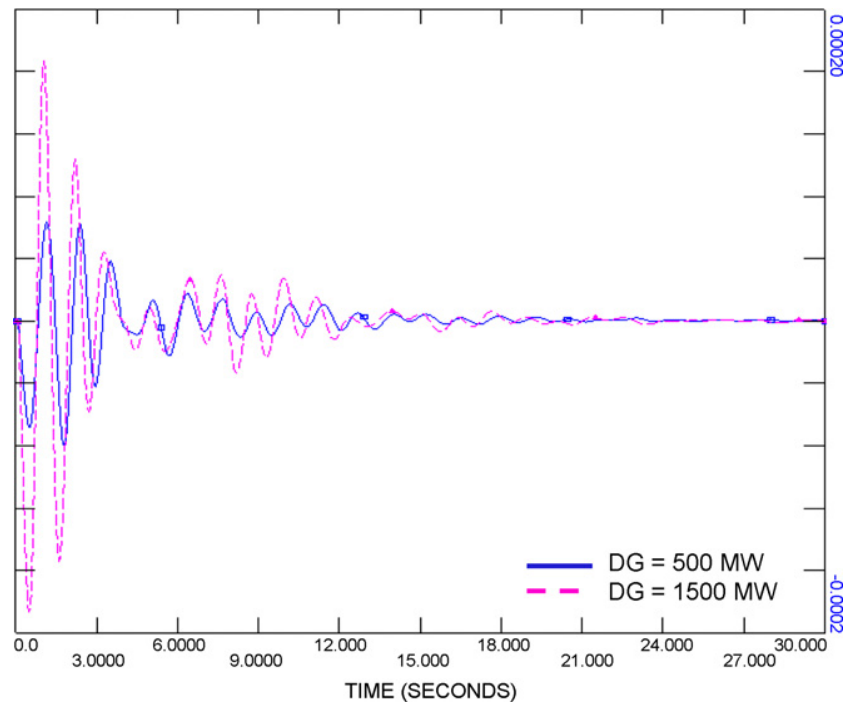

Fig. 6. Frequency behaviour after disturbance 1. 


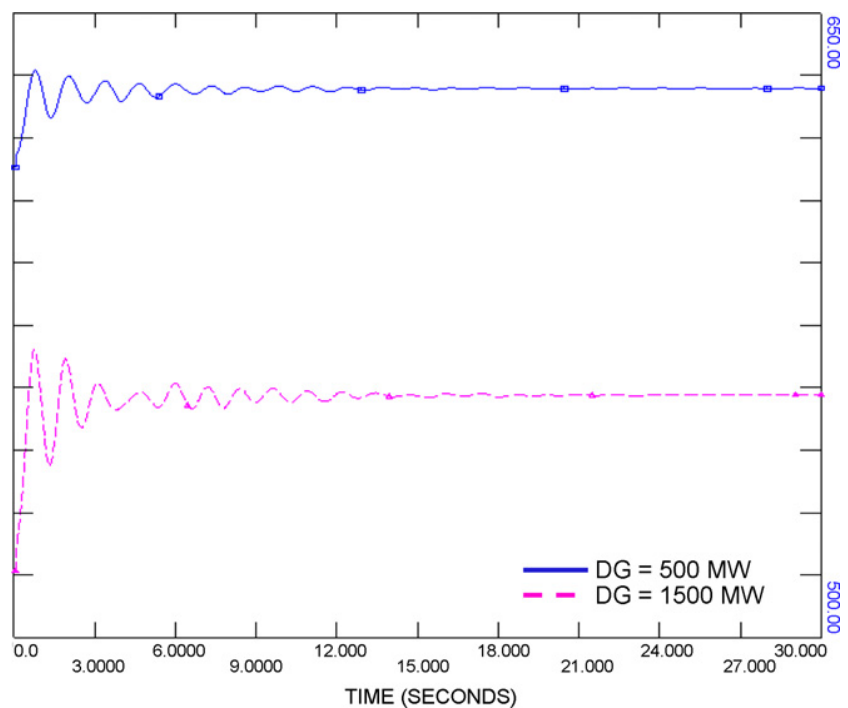

Fig. 7. Power flow behaviour after disturbance 1 in one the remaining $400 \mathrm{kV}$ interconnections.

with $500 \mathrm{MW}$ of DG connected to the grid, whilst the dashed line represents its behaviour for a $1500 \mathrm{MW}$ DG penetration level. These simulations were performed using the PSS/E software.

Since the DG plants do not participate in frequency regulation, one can see from these results that the frequency excursions are larger for the situation where the DG penetration is larger. However, due to the strong interconnection with the European network, the frequency deviations are not very important in absolute value. It is also noticeable from the analysis of Fig. 7 that oscillations in power flow in one of the remaining interconnections are larger and less damped in the scenario characterized by $1500 \mathrm{MW}$ of DG production. These behaviours result from the non-participation of the DG plants in frequency regulation.

In order to illustrate the consequences of the second disturbance, Fig. 8 depicts the system frequency behaviour. In this case the solid line corresponds to the system behaviour with $1500 \mathrm{MW}$ of DG, where $500 \mathrm{MW}$ were lost during the fault elimination period, due to the actuation of the interconnection protections of the DG plants, and the dashed line corresponds to

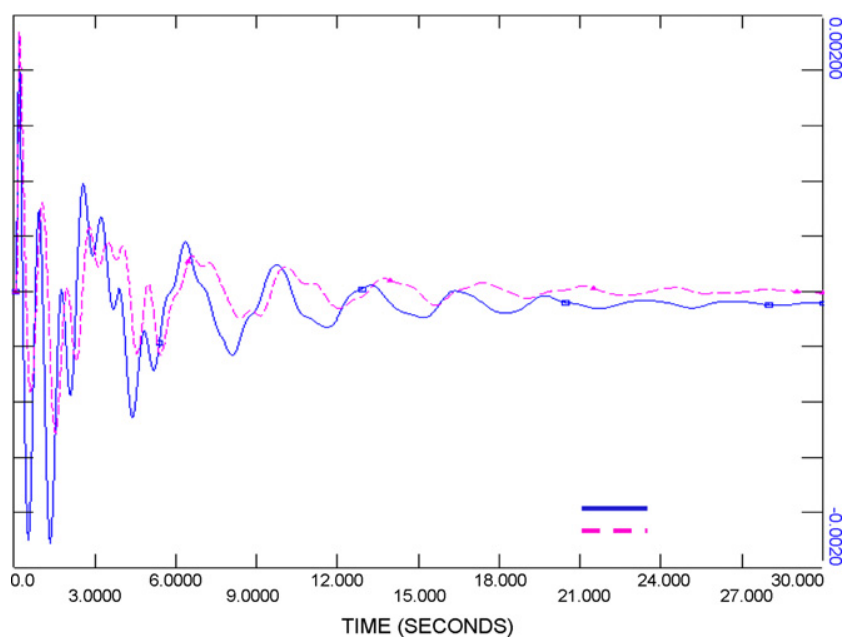

Fig. 8. Frequency behaviour after disturbance 2 .

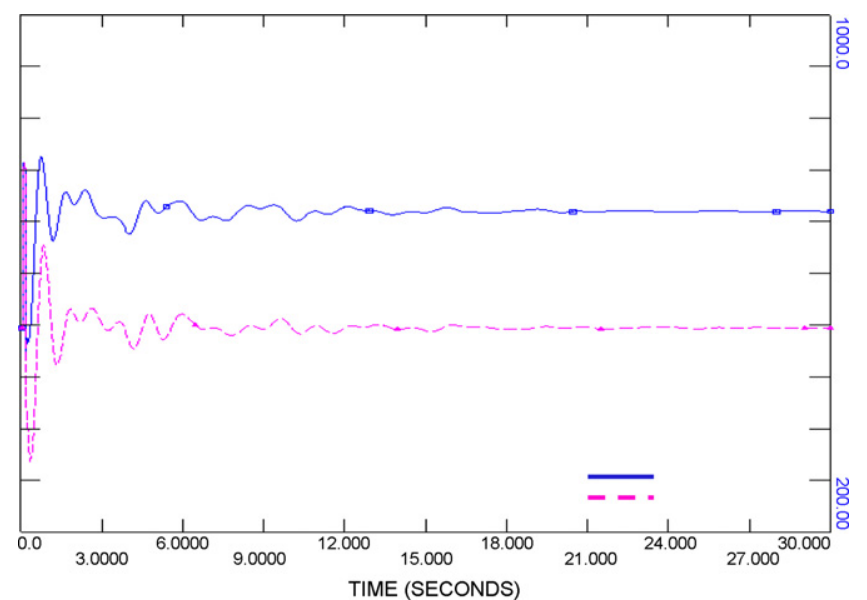

Fig. 9. Power flow behaviour after disturbance 2 in one the remaining $400 \mathrm{kV}$ interconnections.

the system behaviour after a short-circuit in the same location, in a scenario of only $500 \mathrm{MW}$ of DG production situated far from the short-circuit point (and therefore not disconnected from the grid).

From the results described in Fig. 8, one can observe that frequency of the system, for the scenario with larger penetration of DG and disconnection of part of it, reveals, as expected, that frequency stabilized at a different value from $50 \mathrm{~Hz}$. Since a large amount of production in the control area of Portugal was lost, an increase in power import took place $(90 \%$ of the production lost is afterwards imported), as it can be seen from the increase in power flow in one of the interconnection lines (solid line in Fig. 9). The lost DG production is mainly compensated initially from the European network, since the primary reserve regulating capacity of the European system is much larger than the primary regulating capacity of the Portuguese control area. This requires afterwards the activation of the secondary reserves in the Portuguese control area through the AGC operation.

From the analysis of these results, one can conclude that the dynamic behaviour of the system can be strongly affected by the presence of DG units, not only because of its specific behaviour, but also because of the operation of their protection devices or due to the intrinsic nature of the electronic interfaces of the units that use this technology.

Power systems with large shares of hydro production will be able to respond in a very fast and effective way to such type of AGC requests. Systems with pumping reversible units will also be able to deal effectively with these situations. This also means that systems with reduced interconnection capabilities, that may become aggravated in case of large commercial energy trading between control areas, may face serious stability problems in these situations, since such incidents may subsequently provoke a sequence of cascading events leading to the disconnection of the interconnections due to overload conditions. A sudden reduction in wind power production, not properly forecasted, may also lead to overload problems in interconnection lines, which will require in the future the development of area control error (ACE) monitoring tools to identify, in advance, the expected behaviour of the system regarding such incidents. In 
addition, studies on the mobilisation of system load curtailment are required, since these control measures will help in dealing with such situations and will also lead to the effective use of interruptability contracts.

\section{Development of the transmission network}

Planning the expansion of a transmission network in a scenario of large integration of DG requires knowledge about the evolution of the load and the inclusion of estimates on how the DG will grow. These estimates of DG production should be made in terms of energy and capacity to be installed, according to the characteristics of the primary resources available, as well as their geographic distribution. This will enable the identification of conventional production needs, having in mind the availability of the DG resources and system reserves requirements (secondary and tertiary reserves).

The development of the DG demands the availability of a network to receive the DG output. This can be a difficult matter if the DG development happens to be in remote areas. Two types of uncertainties can be identified when solving this problem: (a) the location of the DG plants; (b) the level of expected energy and power to be produced. The definition of a planning strategy for the expansion of the transmission grid requires therefore a certain degree of desegregation, at a regional level, in terms of the possible location of the DG production. The use of geographic information systems, together with models that describe the resources availability and complementary economic and environmental models, can be used to identifying the regional areas where DG production becomes attractive and could therefore appear and require connections to the grids.

Different planning alternatives need then to be considered, characterised by investments costs and robustness indices (such as non-delivered energy, losses, repressed demand and repression on connection of DG). The transmission expansion solution to be adopted should therefore be taken from the analysis of the attributes of these alternative solutions. A deeper discussion of this issue is, however, out of the scope of this paper.

\section{Opportunities for increasing DG penetration}

Experience in the last decade has shown that in order to accelerate the connection rate of DG, be it conventional or renewable, it is necessary to either provide incentives to renewable DG developers and network operators or alternatively to mandate the connection of renewable DG under a regime of preferential feed in tariffs (the German case). The primary reason for support to renewable DG technologies is that for various legitimate reasons they are still relatively expensive sources of energy and moreover tend to disrupt the normal way that power systems are used to operating. And yet they are desirable technologies that must be supported because they are good for the environment and also contribute to sustainability and energy security objectives. In the long term, there is no doubt that these technologies will become competitive as the price of fossil fuels rise due to ever growing demand worldwide for these fuels especially in the growing economies in Asia (China and India). Already there is a strong body opinion suggesting that demand for oil is likely to outstrip supply in the near future. A sustainable energy future therefore cannot be predicated on fossil fuels and must rely on new and renewable technologies. Many countries in Europe now have some form of incentive scheme to support the uptake of renewable sources of energy. There is clearly a lot more that must be done to support DG in general especially from the perspective of integrating DG in the planning and operation of transmission and distribution networks. Some of the key opportunities in this regard are discussed below drawing examples from the UK electricity market.

\subsection{Government renewables incentives}

Some form of renewable incentive schemes are in existence in UK, Spain, Germany, Sweden, Netherlands, Norway and many other EU countries. Most of the incentives benefit distributed renewable generation.

In April 2002, a renewable obligation was introduced in the UK for electricity suppliers where they have to supply a specified percentage of their energy from renewable sources (in 2001-2002, this was 3\%). This can be fulfilled by purchasing renewable obligation certificates (ROCs) from accredited generators. ROCs can be sold separately from the energy. Suppliers that are unable to fulfil their obligation are required to pay a 'buy-out' price to OFGEM for part or the entire Obligation. In 2002, the 'buy-out' price was set at £30/MWh until April 2003. This is adjusted by OFGEM based on the retail price index each year. In 2002, the value of ROCs appears to be comparable to the value of energy sold and as such, provides large financial benefits to renewable generation. The money obtained from the buy-out fund is then recycled to suppliers based on the percentage of ROCs purchased. Being a market based mechanism the value of ROCs will depend on the demand for them, which is turn driven by the extent to which the target has been attained.

In Spain, income from environmental incentives comes from a combination of renewable energy premium, subsidies and tax concessions. A new National Energy Plan ("Plan Energético Nacional") has been prepared and presented in September 2002, which will guide the energy policy for the period up to year 2011. Sustainability is likely to be the focus of the policy driven also by reduction of $\mathrm{CO}_{2}$ emissions.

\subsection{New security standards recognising DG contribution}

A survey of electricity markets in Europe indicates that apart from the UK the majority of countries do not have explicit welldefined security standards. Such standards are important as they drive network investment as will become clear in the following review of the role of security standards in the UK.

Prior to privatisation, the Electricity Council was responsible for setting and maintaining a range of common technical and economic guidance documents, including Engineering Recommendation P2/5. ER P2/5 was intended to be used as a guide to system planning and design. The fundamental principle outlined within ER P2/5 is that there should be sufficient capacity in the system such that, in predefined outage situations, cus- 
tomers continue to receive a supply or have it restored within an acceptable time period. ER P2/5 defines the network design philosophy and, in the context of network pricing, the requirements to comply with the security standard are a key network cost driver. Historically, the structure of electricity distribution networks was driven by an overall design philosophy developed to support large-scale generation technologies. The level of security in distribution networks is defined in terms of the time taken to restore power supplies following a predefined set of outages. Consistent with this concept, security levels on distribution systems are graded according to the total amount of power that can be lost. In general, networks have been specified according to a principle that the greater the amount of power which can be lost, the shorter the recommended restoration time. This philosophy is formalised in the ER P2/5, and this standard is a part of the distribution network licence conditions.

ER P2/5 contains two tables. Table 1 states the minimum demand that must be met after certain specified circuit outages. This level is dependent on the group demand or class of supply. The amount of demand that can be supplied depends upon the available circuit capacities and critically, the contributions from local distributed generators. Table 2 in ER P2/5 specifies the contribution that can be attributed to generation connected to a particular load group.

Given that ER P2/5 was developed in 1970s, it does not currently recognise many of the modern forms of distributed generation presently being connected into distribution networks in the pursuit of Government climate change targets. Consequently, it is not possible to recognise the security contributions of many new forms of distributed generation. It is anticipated that ER P2/6 will supersede ER P2/5 in the near future. This will facilitate the inclusion of security contributions from distributed generation during network planning [20]. In other words, the ER P2/6 will specify the critical condition for network design in the presence of distributed generation.

The new ER P2/6 will provide a basis for quantifying the contribution that DG makes to system security, i.e. the extent to which DG can reduce the demand for network facilities and substitute for network assets. In the network planning stage, generation contribution to network security, as specified by the standards, can be interpreted as firm generation output available at peak demand (during specified period of time for intermittent generation). For various generation technologies, this is specified through a series of contribution factors that take into account the number of generators, their availability and operating regimes. For example, a CHP generator (spark ignition) with an availability of $60 \%$ can contribute to network security with $40 \%$ of its installed capacity.

This is a pre-requisite for establishing cost reflective pricing in networks with DG that can recognise the positive impact that DG may have on network expenditure (reduction of network investment cost) and that can reward generators accordingly.

\subsection{Incentives to DNOs to connect DG}

It has been recognised for sometime now that distribution network companies generally do not have an incentive to connect
DG. In practice DG is in fact viewed as a nuisance disrupting established operating and planning practices with little gain for the network operators. Moreover in the UK, for example, after paying an initial connection charge (based on the deep connection charge policy), generators are exempt from paying ongoing distribution use of system (DUoS) charges. This scenario benefits neither the network operator nor DG. DG, for example is not recognised for the positive impact it has on losses, investment deferral and better utilisation of existing assets. In acknowledgment of this unsatisfactory situation in the UK, a framework has been created within which network companies are incentivised to connect distributed generators based on capacity of DG connected and level of DG utilisation.

\subsection{Review of methodology for distribution Use of system charges}

As indicated above the current regime for DUoS charges does not deal adequately with the presence of DG. The UK Regulator of Gas and Electricity Markets (Ofgem) is in the process reviewing the methodology applied to calculate DUoS charges. There is a desire to develop a DUoS calculation methodology that will recognise the presence of DG and its contribution to system security as well as to losses. The methodology is likely to be based on shallow connection charge policy and have economic efficiency as its key driver.

This review clearly is an opportunity to improve the viability of DG by treating it in a fair and consistent manner recognising the costs/benefits that DG brings to the system within the use of system pricing structure. The approach being taken in the UK can be used by other countries in the EU and elsewhere in the review of their own distribution network pricing methodologies.

\section{Conclusions}

This paper has presented an overview of the key issues concerning the integration of distributed generation into electric power systems that are of most interest to the stakeholders (power system planners and operators, policy makers and regulators, DG developers and customers) in the electrical energy supply industry today. A detailed discussion has been presented of the main drivers of DG integration in many countries around the world. These issues are still just as important and compelling today, perhaps even more so, as they were more than a decade ago when DG became recognised as an important issue in electricity supply. An overview of the main challenges that must be overcome in the integration of DG into electricity supply systems has also been presented. In this paper, particular emphasis was placed on the need to shift network planning and operating policies away from the fit and forget policy of connecting DG to electric power systems to a new more appropriate policy of integrating DG into power system planning and operation though active management of distribution networks. Some of the opportunities that could be exploited in support of the integration and hence greater penetration of DG into electric power systems are also explored. 
The discussion of the impacts of large DG integration in the transmission network shows that there are no dramatic problems for system operation. However, as the DG penetration rises, changes in the operation policy of the network are needed in order to reduce the magnitude of some new problems. The needed operational changes are in the areas of coordination of protection systems and coordination of operation regarding ancillary services. Investments in communication infrastructures and in development of new tools for EMS and DMS environments are needed to support the required changes.

In areas where the DG concentration is large it is recommended to allow generation curtailment, in general for only limited periods of time, in order to comply, when necessary, with the transmission system security limits of operation. However, management of DG unit capabilities should be used also to help manage the local distribution grids and the production/transmission system through the already mentioned additional functionalities of DMS or through the introduction of local production dispatch control centres, able to provide:

- capability of aggregation of hourly DG production levels, in order to provide power forecasts;

- ability to limit production injections in the transmission grid through local DG control;

- ability to limit DG production ramping rates, if necessary;

- management of reactive power support (presently already technically feasible);

- management of active power reserves, thus enabling reductions in secondary reserves (which can be easily implemented in the present technology scenario).

As far as wind power is concerned and when production curtailment is foreseen, it will be necessary to develop combined generation/storage solutions, where the electrical wind power production curtailed for the network could be stored (producing for instance hydrogen or pumping water to upper level reservoirs) and later injected in the system during periods of low wind speeds. Naturally, such capabilities need to be remunerated as reserve services.

Also grid codes need to be revised, having in mind the operational changes that the introduction of large shares of DG will require in the system.

If located in large industrial and residential areas, DG has significant benefits as it decreases the need for building new lines and installing new transformers. In rural areas served by weak grids the increase in DG will require additional investments in transmission infrastructures. These investments have to be decided carefully, having in mind the type of line scheduling that may result from the intermittent nature of the power produced by these units (especially wind generation).
It is also important to mention that the development of DG in remote areas cannot by made on the basis of firm transmission services, where the generating plants must reserve transmission in advance and pay for it independently of the use that is made, as it happens in some systems. Such an approach undermines the development of DG, especially the plants that exploit intermittent renewable primary energy sources.

\section{References}

[1] CIRED Working Group WG04, Dispersed Generation, June 1999.

[2] CIGRE Working Group WG 37-23, Impact of increasing contribution of dispersed generation on the power system, 1997.

[3] COM (97) 559, Communication from the European Commission: energy for the future, renewable sources of energy, White Paper for a Community Strategy and Action Plan.

[4] European Commission, Directive of the European Parliament and the Council on the Promotion of Electricity from Renewable Energy Sources in the Internal Electricity Market, 2000.

[5] N. Hatziargyriou, A. Zervos, Wind power development in Europe, Proc. IEEE 89 (12) (2001) 1765-1782.

[6] Wind Directions, vol. XXII, No. 1, November 2002.

[7] Ofgem, Electricity distribution price control review Appendix-Further details on the incentive schemes for distributed generation, innovation funding and registered power zones, June 2004 http://www.ofgem. gov.uk/ofgem/work/index.jsp?section=/areasofwork/distpricecontrol.

[8] N.D. Hatziargyriou, T.S. Karakatsanis, M. Papadopoulos, Probabilistic load flow in distribution systems containing wind power generation, IEEE Trans. Power Syst. 8 (1) (1993) 159-165.

[9] N. Hatziargyriou, T. Karakatsanis, G. Strbac, Connection criteria for renewable generation based on probabilistic analysis, in: Sixth International Conference on Probabilistic Methods Applied to Power Systems, PMAPS'2000, Funchal, Madeira, Portugal, September 25-28, 2000.

[10] EC Contract JOR3-CT98-0201, Final Report, Electricity Tariffs and Embedded Renewable Generation, July 2000.

[11] N. Jenkins, R. Allan, P. Crossley, D. Kirschen, G. Strbac, Embedded Generation, IEE Power and Energy Series 31, London, 2000.

[12] Ilex Energy Consulting with the Manchester Centre for Electrical Energy, UMIST, A report for DTI on Ancillary Service Provision from Distributed Generation, September 2004, URL: http://www.dti.gov. uk/renewables/publications_pdfs/dgcg000300000.pdf.

[13] J. Peças Lopes, Integration of dispersed generation on distribution networks - impact studies, in: Proceedings of the IEEE Winter Meeting, N.Y., February 2002.

[14] P. Smith, H.K. Clark, Impact of increasing wind generation on the transmission system in the Republic of Ireland, in: Proceedings of the CIGRE Symposium Neptune 1997, Romania, 1997.

[15] T.P. Leão, M.A. Matos, Distribution Planning with Fuzzy Loads and Independent Generation, in: Proceedings of the 14th International Conference and Exhibition on Electric Distribution, 1997.

[16] P. Kundur, Power System Stability and Control, McGraw-Hill, 1993.

[17] J.G. Slootweg, S. de Haan, H. Polinder, W. Kling, Modeling new generation and storage technologies in power system dynamics simulations, in: Proceedings IEEE Summer Meeting, Chicago, July 2002.

[18] J. Machowski, J. Bialek, J. Bumby, Power Systems Dynamics and Stability, John Wiley \& Sons, 1997.

[19] J.G. Slootweg, W. Kling, Impacts of distributed generation on power system transient stability, in: Proceedings of the IEEE Summer Meeting, Chicago, July 2002.

[20] R. Allan, G. Strbac, P. Djapic, K. Jarrett, Methodology for developing ER P2/6, DTI Report, April 2004 